Ebisu Ebisu

Études japonaises Études japonaises

$58 \mid 2021$

2011-2021 : crises, ruptures et nouvelles dynamiques.

Dix ans après la triple catastrophe du 11 mars

\title{
Fukushima en procès : un mouvement social dans les tribunaux pour repenser la catastrophe
}

フクシマ訴訟：炎害を再考察するための法廷における社会運動

Fukushima on Trial: a Social Movement in the Courts to Rethink the Disaster

\section{Paul Jobin}

\section{OpenEdition}

\section{Journals}

Édition électronique

URL : https://journals.openedition.org/ebisu/6118

DOI : $10.4000 /$ ebisu. 6118

ISSN : 2189-1893

Éditeur

Institut français de recherche sur le Japon à la Maison franco-japonaise (UMIFRE 19 MEAE-CNRS)

\section{Édition imprimée}

Date de publication : 1 novembre 2021

Pagination : 299-324

ISSN : 1340-3656

\section{Référence électronique}

Paul Jobin, «Fukushima en procès : un mouvement social dans les tribunaux pour repenser la catastrophe », Ebisu [En ligne], 58 | 2021, mis en ligne le 01 novembre 2021, consulté le 13 avril 2022. URL : http://journals.openedition.org/ebisu/6118; DOI : https://doi.org/10.4000/ebisu.6118 


\section{Fukushima en procès : un mouvement social dans les tribunaux pour repenser la catastrophe}

Paul Jobin

フクシマ訴訟：災害を再考察するための法廷における社会運動

$$
\text { ポール・ジョバン }
$$

Fukushima on Trial: a Social Movement in the Courts to Rethink the Disaster Paul Joвın

\begin{abstract}
\ots-clés : Fukushima, nucléaire, actions collectives, action en justice, procès, mobilisation, radiations
\end{abstract}

L'auteur: Paul Jobin est chercheur à l'Institut de sociologie, Academia Sinica, à Taiwan. Il a mené des recherches sur les travailleurs des centrales nucléaires à Fukushima avant et après la catastrophe nucléaire de mars 2011.

Résumé : Comme les nombreuses catastrophes industrielles qui ont marqué l'histoire du Japon moderne et contemporain, la catastrophe nucléaire de mars 2011 a donné lieu à de nombreux procès. Les procès de Fukushima réunissent plusieurs milliers de plaignants à travers tout le Japon. Outre un procès pénal contre trois anciens dirigeants de Tepco, on relève une trentaine de plaintes collectives au civil contre Tepco et l'État. Ces procès posent des questions fondamentales sur l'origine de cette catastrophe et, compte tenu du problème des radiations, son devenir. Et bien que les montants d'indemnisation retenus par les juges jusqu'à présent restent faibles, ces décisions de justice revêtent pour les plaignants une importance morale et symbolique (voire des effets cathartiques), ainsi que pour le Japon contemporain, et à l'avenir pour d'autres pays qui pourraient être touchés par une catastrophe nucléaire. 


$$
\text { キーワード }
$$

福島、原発事故、原子力、団体訴訟、訴訟運動、 民事訴訟、刑事訴訟、放射能

\section{著者}

ポール・ジョバン：台湾・中央研究院社会学 研究所研究員。2011 年 3 月の原発事故前後 の福島の原発作業員に関する研究を行った。

\section{要旨}

2011 年 3 月の原発事故は、近現代の日本に おける多くの産業災害と同様に、数多くの訴 訟を引き起こした。今日、フクシマ訴訟のた
めに、日本全国で数千人の原告が結集してい る。東京電力の旧経営陣 3 人に対する刑事 訴訟に加えて、東京電力及び国に対する民事 集団訴訟は約 30 件に上る。これらの裁判は、 この災害の原因、そして放射能という問題を 考慮して、その将来的な影響についても本質 的な問いを投げかけている。現在まで裁判官 によって言い渡された賠償金額が僅かであっ たとしても、これらの判決は、原告及び現代 日本、そして将来的に原子力災害で被害を受 ける可能性のある諸外国にとつても、倫理的 そして象徴的に重要である。
Veywords: Fukushima, Nuclear, Class Actions, Lawsuits, Mobilisation, Radiations

The Author: Paul Jobin is a researcher at the Institute of Sociology, Academia Sinica, in Taiwan. He conducted research on nuclear power plant workers in Fukushima before and after the nuclear disaster of March 2011.

Abstract: Like many industrial calamities that have marked the history of modern and contemporary Japan, the March 2011 Fukushima nuclear disaster has given rise to many lawsuits; currently, these involve several thousand plaintiffs from all over Japan. On top of a criminal case against three Tepco executives, there are more than 30 class actions pending against Tepco and the state. These lawsuits raise fundamental questions about the origin of the disaster as well as its future fallouts due to radiation exposure. While the compensation awarded by the judges so far remains low, the court decisions carry a moral and symbolic-even cathartic-importance for the plaintiffs, as well as for contemporary Japan as a whole and for other countries vulnerable to a nuclear disaster. I further argue that these mobilisations are drawn from a legacy of collective lawsuits that have marked Japan over the past 50 years. 


\section{Fukushima en procès : un mouvement social dans les tribunaux pour repenser la catastrophe}

Paul JoBIN*

Comme les nombreuses catastrophes industrielles qui ont marqué l'histoire du Japon moderne et contemporain, la catastrophe nucléaire de mars 2011 a donné lieu à un grand nombre de litiges. Outre un procès pénal (keiji soshō 刑事 訴訟) dont l'initiative revient à un mouvement citoyen, à travers tout le Japon, on relève une trentaine d'actions collectives au civil (minji soshō 民事訴訟) et deux auprès du tribunal administratif (gyōsei soshō 行政訴訟). L’ensemble des actions au civil réunit plus de douze mille demandeurs. En première instance, dix-neuf tribunaux de district (chihō saibanshō 地方裁判所) ont déjà rendu leurs verdicts, dont une grande majorité en faveur des demandeurs contre Tepco (Tokyo Electric Power Company) et l'État japonais, et ces actions se poursuivent maintenant devant les cours d'appel (kōtō saibansho 高等裁判所).

Note de l'auteur : Pour conduire la recherche qui préside à cet article, j’ai bénéficié d'une invitation, en novembre 2019, de l'Institut français de recherche sur le Japon à la Maison franco-japonaise (IFRJ-MFJ) de Tokyo. Je remercie Bernard Thomann, Rémi Scoccimarro et Anne Gonon pour cette invitation, ainsi que les participants du séminaire qui s'est tenu à la MFJ le 16 novembre 2019, pour leurs remarques stimulantes sur une première version de ce texte. Je suis également redevable à Kojima Rina pour son aide dans la prise de contact avec les plaignants qui ont accepté d'être interviewés. Cet article est une traduction librement adaptée de l'anglais et actualisée d'un article paru en mai 2020 dans la revue en ligne The Asia Pacific Journal: Japan Focus. Je remercie les éditeurs de ce numéro, le comité de rédaction et le lecteur anonyme pour leurs remarques et corrections très utiles.

* Chercheur à l'Institut de sociologie, Academia Sinica (Taiwan). 
Contrairement à la politique de réparation et les diverses mobilisations sociales, les procès qui ont fait suite à la catastrophe de Fukushima ont fait l'objet de peu d'études, hormis des articles ou des reportages dans la presse. Ils posent pourtant des questions fondamentales sur l'origine de cette catastrophe et, compte tenu du problème des radiations, son devenir.

Dans cet article, je souligne l'importance symbolique du procès pénal, les difficultés rencontrées par les demandeurs dans ces actions au civil, et je montre qu'en dépit des montants faibles d'indemnisation, ces procès n'en sont pas moins très importants pour le Japon contemporain, ainsi que pour d'autres actions portant sur des dommages industriels et technoscientifiques. Je soutiens en outre que ces mobilisations s'appuient sur un legs d'actions collectives qui se sont déroulées au Japon au cours des cinquante dernières années et aux caractéristiques originales. Outre les études, d'une part sur la catastrophe nucléaire de Fukushima, et d'autre part sur l'interaction entre droit et société au Japon, je prends appui sur des entretiens conduits avec des représentants de groupes de plaignants et sur des documents qu'ils ont mis à ma disposition. La section suivante présente quelques aspects essentiels des contextes juridique et politique.

\section{Le contexte juridique et politique}

Le taux relativement faible au Japon de différends portés au tribunal a longtemps été attribué à des barrières culturelles ou institutionnelles. La comparaison vaut surtout avec les États-Unis où le recours aux services d'un avocat est très ancré dans la vie quotidienne. De nombreux universitaires japonais ont déployé une version juridique des visions culturalistes du Japon (Nihonjinron 日本人論), arguant de la propension culturelle des Japonais à éviter les litiges pour valoriser le consensus et l'harmonie, jusqu'à ce que des chercheurs américains comme Frank Upham $(1976,1987,2005)$ ou John Haley $(1978,1991)$ ne mettent en pièce cette interprétation culturaliste.

Plusieurs réformes importantes ont facilité l'accès aux tribunaux : une refonte du code de procédure civile (en 1996), l'adoption (en 1999) de la loi sur l'accès à l'information et un remaniement de l'ensemble du système judiciaire (Foote 2014 : 174-180). Ces réformes ont permis d'augmenter le nombre de juges, de procureurs et d'avocats, et à la différence du puissant machisme qui persiste dans d'autres professions, la proportion de femmes a 
sensiblement augmenté 1 . Cette évolution facilite notamment les demandes de réparations qui nous occupent ici.

L'indépendance du pouvoir judiciaire japonais a également fait l'objet d'un long débat comparatif avec les États-Unis (Haley 1998; Ramseyer \& Rasmusen 2003). Pour ce qui concerne les procès de Fukushima, même si les différents acteurs intervenant dans ces procès tendent plutôt à éviter le sujet (à commencer par les juges, bien sûr, mais aussi les avocats, les plaignants et les médias), ces procès ont forcément des implications politiques. En premier lieu vient bien sûr la question du redémarrage des centrales. Contrairement aux ex-premiers ministres Kan Naoto 菅直人 (2010-2011) et Koizumi Jun.ichirō 小泉純一郎 (2001-2006), qui sont devenus de fervents opposants à l'énergie nucléaire après la catastrophe, Abe Shinzō 安倍晋三 (deuxième mandat : 2012-2020) a constamment affirmé sa volonté de redémarrer le plus grand nombre possible de centrales. Or, la situation dans la centrale de Fukushima Daiichi est loin d'être résolue. Selon le gouvernement lui-même, il faudra au moins trente ou quarante ans de plus pour "nettoyer " l'ensemble du site, voire jusqu'à deux cents ans selon d'autres estimations. Des robots ont été utilisés pour inspecter les réacteurs endommagés, mais les niveaux de radiation restent tellement élevés, qu'ils sont toujours pratiquement inutiles pour les opérations de nettoyage.

Malgré l'opposition des syndicats de pêcheurs, Tepco et le gouvernement souhaitent rejeter dans l'océan les centaines de cuves d'eaux contaminées au tritium et autres radionucléides. En outre, le site de la centrale et ses environs sont aussi devenus une immense zone de stockage pour la "décontamination " des terrains; une autre tâche de Sisyphe consistant à accumuler du fumier contaminé sous des kilomètres de bâches plastiques qui finissent par devenir poreuses, mais qui ne sont pas remplacées. Pour Tepco et le gouvernement, jusqu’à présent, il s'agissait moins de remédier à la contamination durable des sols, des produits agricoles et de la pêche, que d'encourager un retour à la normale, au moins en apparence. Le gouvernement n’a cessé de sous-estimer le risque de contamination dans la région de

1. Le nombre de juges et de procureurs est passé respectivement de 2143 et 1363 en 1999 à 2774 et 1976 en 2019, tandis que le nombre d'avocats a fait un bond important, de 16731 à 41118 . Quant aux femmes, elles représentent aujourd'hui environ un quart des trois professions : $19 \%$ des avocats, $25 \%$ des procureurs et $27 \%$ des juges (Nichibenren 2019). 
Fukushima et ses conséquences sur les approvisionnements alimentaires à travers tout le Japon.

Mais dans les procès de Fukushima, ce sont surtout les déplacements de population qui occupent une place centrale. Selon les données de l'État, telles que celles publiées par l'Agence japonaise de reconstruction, la catastrophe nucléaire elle-même a provoqué l'évacuation d'environ 164000 personnes avant de diminuer progressivement pour atteindre environ 79000 personnes (Xuan 2019). Fin mars 2017, le gouvernement a réduit l'aide publique à 27000 personnes déplacées par la catastrophe (Kojima 2020). Seuls $10 \%$ des évacués sont retournés dans leurs maisons abandonnées, en majorité des personnes âgées.

Le 11 mars 2020, neuf ans après la catastrophe nucléaire, la une de l'Asahi shinbun 朝日新聞 a déploré le manque d'intérêt pour cette situation, même parmi les habitants de Fukushima eux-mêmes (Kikuchi 2020). Autre signe des temps, sur la photo qui accompagne l'article, les secouristes rendant hommage à leurs collègues décédés en 2011 portent des masques, non pour empêcher les radiations, mais à cause de la pandémie de Covid-19.

Avec le règlement extrajudiciaire des litiges instauré par l'État (saibangai funsō kaiketsu tetsuzuki 裁判外紛争解決手続き), les victimes peuvent demander une indemnisation pour des dommages que Tepco refuse de payer (Kojima 2020). L’objectif de Tepco et de l'État est de réduire le nombre de batailles juridiques. Mais ce système n’a pas éliminé la frustration. Si le nombre total de demandeurs ne représente qu'une infime partie de tous ceux dont la vie a été bouleversée par la catastrophe, pour le gouvernement pressé de relancer les centrales nucléaires, leur action gêne. Comme l'exprime Maeda Akira 前田朗, le leader d'un groupe de demandeurs à Yokohama, les principales victimes de la catastrophe expriment à travers les procès de Fukushima contre l'État et Tepco leur refus de se voir reléguer comme des citoyens de seconde zone (kimin 棄民) (Maeda et al. 2019 : 63). Ou bien, comme l'exprime Morimatsu Akiko, à la tête du groupe des demandeurs à l'origine de l'action engagée auprès du tribunal de district d'Osaka :

Le Premier ministre Abe et son gouvernement ont envoyé de nombreux signaux indiquant qu'avant 2020, il fallait éliminer le nombre de victimes officielles de la 
catastrophe nucléaire. Nous sommes un fardeau pour le gouvernement et une tache dans le paysage des Jeux olympiques².

Les Jeux olympiques de 2020, reportés en 2021, ont été présentés à la région de Fukushima comme une opportunité pour relancer la croissance économique et restaurer la confiance nationale. Ce volontarisme motivé par les Jeux olympiques - sorte de méthode Coué de la gestion d'un drame nucléaire à long terme - jette un voile pudique sur l'immensité du chantier qui reste à accomplir à Fukushima Daiichi. Mais le pire est sans doute que le fardeau de la catastrophe est mis sur les épaules des victimes, par des appels à la " résilience " (Scoccimarro 2016; Ribault 2019; Kojima 2020), ou bien, comme l'expriment plus haut Maeda et Morimatsu, par une injonction à disparaître du paysage.

Pour le groupe de citoyens qui a initié le procès pénal, ainsi que pour tous les demandeurs à l'origine des actions collectives au civil, le verdict du procès pénal de septembre 2019 a été perçu comme une énorme injustice très influencée par le contexte politique (Johnson et al. 2020). Beaucoup avaient espéré qu'une sanction contre les trois dirigeants de Tepco enverrait un signal fort au gouvernement pro-nucléaire d'Abe. Pour Johnson et al, il est probable que la bataille au pénal dure encore plusieurs années avant d'être finalement rejetée par la Cour suprême. De sorte que les actions collectives au civil à travers tout le pays prennent en quelque sorte le relais du procès pénal sur le plan politique, pour obtenir des réparations, exiger une reconnaissance symbolique de la catastrophe et un arrêt définitif de toutes les centrales nucléaires.

\section{Motivations des demandeurs et décisions de la cour}

Toutes les parties à l'origine des actions collectives au civil demandent une indemnisation, soit seulement à Tepco (quatre sont dans ce cas), soit à la fois à Tepco et à l'État (vingt-sept cas), en particulier pour les dommages matériels, tels que la perte d'un logement ou d'une entreprise, et les conséquences connexes, telles que la détresse psychologique ${ }^{3}$.

2. Entretien à Osaka, 14 novembre 2019.

3. Pour un tableau synthétique de tous ces procès, voir Jobin (2020) et Yokemoto (2020), et pour un suivi : fukushimagenpatsu.bengodan.jp. 
Au 30 septembre 2020, dix-sept jugements avaient été rendus en première instance, et trois arrêts en appel. Seize tribunaux ont ordonné à Tepco de verser des réparations aux demandeurs et dans sept cas, l'État a également été jugé responsable. Dans un seul cas (Yamagata, 17 décembre 2019), les juges ont rejeté les demandes contre Tepco et l'État. Dans tous les autres cas, les tribunaux ont marqué leurs distances avec les normes établies par le dispositif de conciliation extra-judiciaire de l'Etat pour l'indemnisation des dommages de l'industrie nucléaire (genshiryokusongaibaishōfunsōshinsa-kai 原子力損害賠償紛争審査会). On peut donc dire que, sur le fond, les demandeurs obtiennent généralement gain de cause et que le bilan général de ces procès leur est favorable.

Le délai entre la saisine du tribunal et le jugement varie de quatre à six ans. Bien que cela puisse sembler long, c'est à peu près la durée moyenne pour ce type d'affaires au Japon. Cependant, dix-sept actions sont encore en attente de jugement en première instance. Malgré la condition précaire des personnes déplacées par la catastrophe nucléaire, les tribunaux n’ont pas pris de mesure particulière pour accélérer la procédure. Qui plus est, Tepco et l'État ont fait appel de tous les jugements. Au regard des autres grandes batailles juridiques que le Japon a déjà connues, tels que les procès pour les victimes de la bombe atomique, la maladie de Minamata, ou encore les ouvriers atteint par l'amiante, les procès liés à Fukushima vont probablement se poursuivre pendant des années, voire une ou plusieurs décennies.

On peut distinguer deux grandes catégories de poursuites : l'une porte sur la restauration d'un environnement exempt de radiations à Fukushima; l'autre met l'accent sur le droit de recommencer une nouvelle vie ailleurs, en partant du principe qu'il faudra probablement des décennies avant que le danger des radiations ne soit éliminé. Dans le premier cas, les demandeurs souhaitent pouvoir retourner vivre à Fukushima, comme le résument les appels de ralliement tels que "Rendez-nous nos vies antérieures! (Moto no seikatsu o kaese 元の生活を返せ), «Rendez-nous notre source de travail, rendez-nous notre région» (Nariwai o kaese, chiiki o kaese 生業を返せ、 地域を返せ), etc. Ces affaires comptent 6489 demandeurs, soit plus de la moitié des membres de la coalition nationale Gensoren 原訴連 (abréviation de 原発被害者訴訟原告団全国連絡会). Les autres, soient 5920 autres demandeurs engagées dans un total de 26 actions à travers tout le pays (de Hokkaidō à Kyūshū), réclament un soutien financier pour vivre à l'abri des radiations (binan no kenri 非難の権利) et indépendamment des critères du gouvernement en la matière. 
Malgré ces différentes priorités, l'élimination des radiations à Fukushima pour les uns ou le droit de vivre ailleurs pour les autres, tous ces procès partagent des objectifs communs. L'avocat Kurozawa Tomohiro 黒澤知弘, qui dirige le groupe des demandeurs dans le procès de Kanagawa, souligne trois motivations principales (Maeda et al. 2019 : 7-24). La première est de prouver la responsabilité de Tepco et de l'État face au manque de mesures préventives contre les tremblements de terre et les tsunamis; sur ce point, les preuves présentées au pénal ont été d'un grand secours dans les actions au civil. Une autre motivation est de remettre à plat les critères d'indemnisation fixés par l'État et Tepco pour les personnes déplacées par la catastrophe. Enfin, il s'agit de contester les normes de radioprotection revendiquées par l'État jusqu'à présent pour définir les territoires à risque.

Tous les demandeurs ont été chassés de chez eux par la catastrophe, ce qui, selon les normes onusiennes, fait d'eux des réfugiés à part entière (binansha 避難者). Bien que les demandeurs comprennent aussi des " évacués forcés " (kyōsei hinansha 強制避難者), l'État a classé la majorité d'entre eux comme des « évacués volontaires " (jishu hinansha 自主避難者), comme si leur départ était une question de convenance personnelle, indépendamment du risque accru d'exposition radioactive (Kojima 2020). Par conséquent, beaucoup se sont retrouvées exclues du plan d'indemnisation instauré par Tepco et l'État; pour ceux qui, avant la catastrophe, résidaient hors des "zones d'évacuation " (ainsi qualifiées après mars 2011), seuls les enfants, les femmes enceintes et quelques autres exceptions sont autorisés à demander des indemnités, pour des montants de toute façon très modestes. Qui plus est, ce plan d'indemnisation a pris fin en mars 2017, laissant de nombreuses familles dans une situation économique difficile. Ces différentes catégories résultent d'un zonage du risque qui a diminué comme une peau de chagrin alors que les radiations, elles, persistent durablement. Ce zonage territorial (et les catégories qui en découlent) sert malheureusement de cadre de référence aux juges pour le calcul des réparations; ils peuvent toutefois l'interpréter librement.

L'évaluation des conséquences des « faibles doses » d'exposition aux rayonnements est un élément crucial des débats. Le modèle de progression du risque linéaire sans seuil (Linear No-Threshold, LNT) conteste l'existence d'un seuil de sécurité en dessous de 100 millisieverts ( $\mathrm{mSv}$ ), voire en dessous de $20 \mathrm{mSv}$; ce modèle est désormais soutenu par un solide consensus dans la communauté scientifique internationale, ainsi que parmi la plupart des experts au Japon. Mais transformer ces évaluations en normes juridiques pour la santé 
publique est une autre histoire. Après l'accident nucléaire de mars 2011, le gouvernement a adopté $20 \mathrm{mSv}$ comme seuil de sécurité pour l'exposition aux rayonnements sans tenir compte des conséquences (Jobin 2013b; Shirai 2015; Ribault 2019; Jobin 2021). Selon la médecin Sakiyama Hisako 崎山比早子, qui a témoigné dans trois procès à la demande des requérants (Chiba, Kyoto, Tokyo), les experts sollicités par Tepco et l'État ne peuvent pas argumenter contre les conclusions du modèle linéaire sans seuil. Mais ils en atténuent les conséquences, comme si les risques entre 1 et $20 \mathrm{mSv}$ étaient négligeables, ignorant ainsi nombre d'études épidémiologiques récentes montrant que l'exposition à des doses comprises entre 1 et $20 \mathrm{mSv}$ entraîne un risque accru de cancer et de lésions de l'ADN (Maeda et al. 2019: 41-56).

Jusqu’à présent, seuls les juges de Tokyo ont validé explicitement le modèle linéaire sans seuil (décision en première instance, en mars 2018). En plus des actions au civil, deux actions collectives dans le cadre de procès administratifs contre l'État se concentrent sur le problème des normes de rayonnement. En contestant le seuil de sécurité de $20 \mathrm{mSv}$, tous ces procès remettent en cause le zonage territorial fixé au lendemain de la catastrophe et progressivement réduit par la suite pour limiter le nombre de personnes pouvant bénéficier d'une indemnisation.

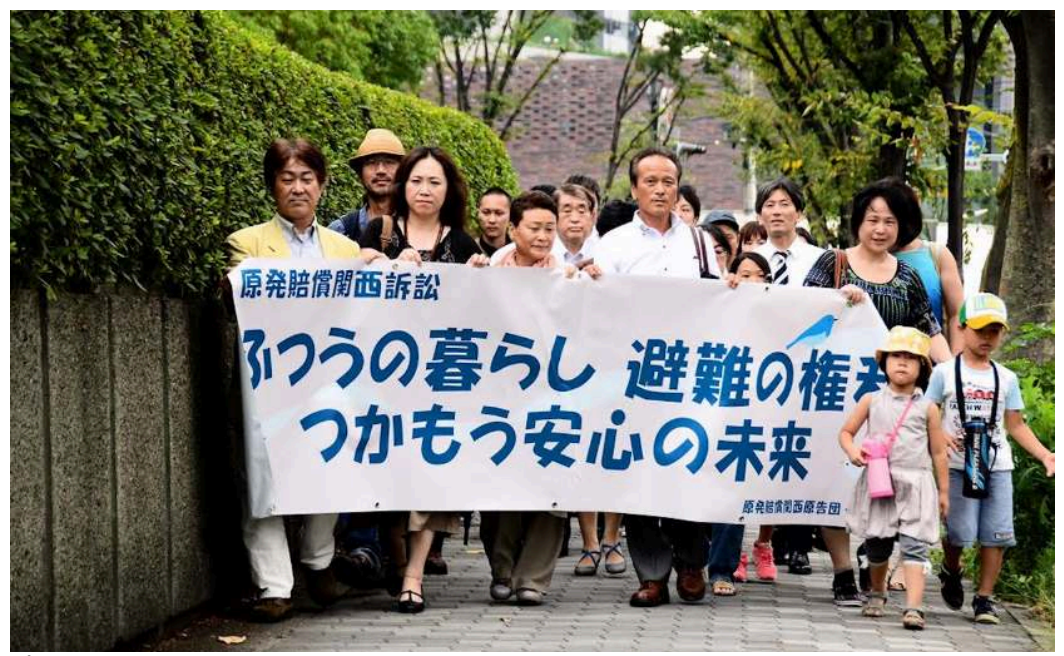

Fig. 01

Les requérants se rendent à une audience au tribunal d'Osaka, 30 juillet 2015.

Source : Akiko Morimatsu. 


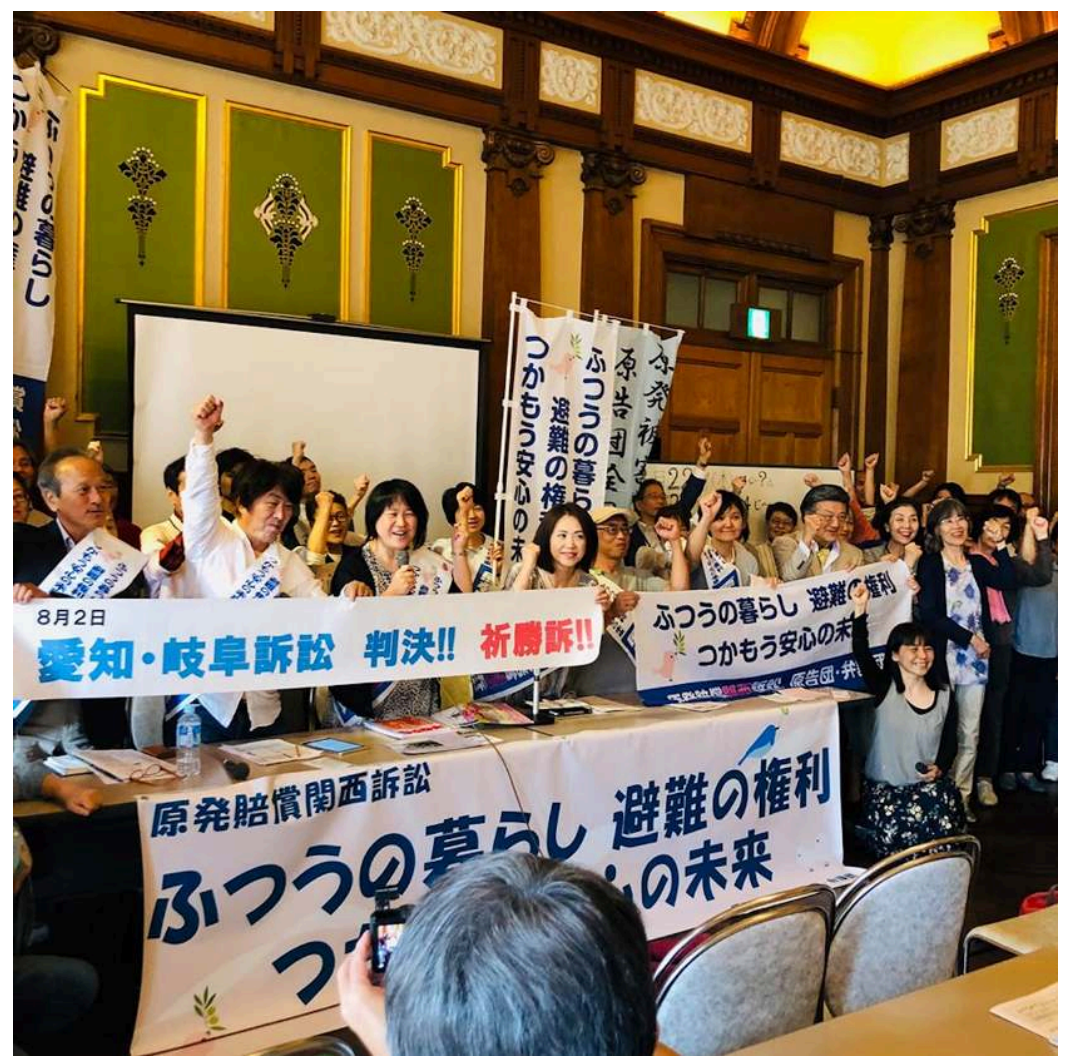

Fig. 02

Réunion des requérants après une audience au tribunal d'Osaka, 23 mai 2019.

Source : Akiko Morimatsu.

\section{Des indemnités faibles}

$\mathrm{Si}$, dans l'ensemble des actions, les juges ont donné raison aux demandeurs sur le fond, en revanche, les montants d'indemnisation sont très décevants. Voyons quelques exemples et leur portée symbolique.

L'une des premières décisions de justice a été rendue en février 2016, et elle ne concernait pas une action collective, mais une famille qui avait quitté la ville de Koriyama (département de Fukushima). Bien que Koriyama soit 
située en dehors de la zone d'évacuation officielle, le tribunal a tenu compte du fait que ses habitants étaient exposés à un niveau de rayonnement naturel dépassant les normes de sécurité. Comme la mère était enceinte et que la famille avait déjà un jeune enfant, ils avaient décidé de déménager à Kyoto. Le père, qui avait un restaurant à Koriyama, a essayé de démarrer une autre affaire à Kyoto, mais, face aux difficultés, il est tombé en dépression, et il a alors engagé une action contre Tepco pour syndrome de stress post-traumatique. En première instance, le tribunal de district de Kyoto a ordonné à la société de lui verser trente millions de yens à titre de compensation. Or, deux ans plus tard, dans le procès opposant un groupe de 58 familles, dont deux familles d'évacués "forcés " et 49 d'évacués " volontaires ", les montants étaient considérablement inférieurs : 600000 yens pour les enfants des personnes évacuées volontairement, et 300000 yens pour les adultes. Si cette tendance se confirmait, elle pourrait nuire à la dynamique d'actions collectives, en encourageant un éparpillement et une individualisation des actions en justice. Mais, pour l'heure, les collectifs de requérants restent solidaires du travail élaboré par leurs avocats.

La première décision dans un procès initié par une action collective a été rendue en mars 2017 par le tribunal de district de Maebashi (Kikuchi 2017). Les demandeurs étaient des réfugiés "forcés " et "volontaires ", dont la plupart avaient quitté des maisons situées à moins de trente kilomètres de Fukushima Daiichi (Soeda 2017 : 101). Ce tribunal a été le premier à reconnaître la responsabilité de l'entreprise et de l'État. Après avoir examiné les témoignages des experts et inspecté les lieux, les juges ont décidé que Tepco et les autorités de l'État avaient, dès 2002, clairement conscience que le système de refroidissement des réacteurs nucléaires pourrait être détruit par un tsunami de plus de dix mètres. Cette décision a créé un précédent crucial pour les autres procès en cours (Soeda 2017 : 110-118). Mais les demandeurs ont été consternés par les faibles montants d'indemnisation fixés par les juges : un maximum de cinq millions de yens pour les familles ayant quitté les zones d'évacuation officielles, déduction faite, le cas échéant, d'une somme de 1,8 million de yens versée par l'État et Tepco dans le cadre du plan d'indemnisation pour la catastrophe. Pour ceux qui ont perdu leur maison et leur travail, de tels montants ne représentent bien sûr qu'une part minime des dommages.

Quelques mois plus tard, en septembre 2017, le tribunal de district de Chiba a ordonné à Tepco de verser un montant d'indemnités analogue au 
jugement de Maebashi. Pire, les juges n'ont pas jugé l'État responsable. La troisième décision, en octobre 2017, concernait le groupe le plus important de requérants ("Rendez-nous notre source de travail " nariwai o kaese) : le tribunal de district de Fukushima a lui aussi approuvé la responsabilité de Tepco et de l'État, mais en rejetant les demandes d'un quart des requérants et en fixant des indemnités très basses (entre 200000 et un million de yens). Fin septembre 2020, la cour d'appel de Sendai a réintégré l'ensemble des 3500 appelants, aggravé les charges contre les intimés, et comme il s'agit de la première décision en appel qui tient compte de la responsabilité de l'Etat, elle influencera sans doute le cours des autres procès (Yokemoto 2020). Mais, même si le montant total a été doublé pour atteindre 101 millions de yens, cela ne fait jamais qu'un peu moins de 300000 yens (environ 2500 euros) en moyenne par requérant, ce qui reste très faible. Jusqu'à présent, les montants les plus élevés ont été attribués par une décision en première instance, en mars 2018, du tribunal de district de Tokyo : 60 millions de yens pour une quarantaine de demandeurs, soit un peu moins d'1,5 million par demandeur en moyenne.

Dans les actions collectives, malgré les difficultés de nombreux demandeurs à joindre les deux bouts, la réparation économique du dommage n'est pas la seule motivation; elle semble même plutôt venir en second plan. Dans de nombreuses luttes similaires à travers le monde, "l'environnement » est souvent perçu comme une part constitutive de la personne et de sa communauté; de sorte que sa traduction juridique en termes de "perte d'usufruit " ne signifie pas grand-chose pour les victimes.

Dans les procès de Fukushima, les demandeurs expriment ce sentiment par des mots d'ordre tels que « la perte du pays natal » (furusato no sōshitsu 故郷の整失), c'est-à-dire la disparition d'une histoire et d'une culture communes. À défaut, certains déplacés se retrouvent sur le lieu de l'exil, et les procès contribuent à renouer ces liens communautaires. Mais, comme l'a souligné Yokemoto Masafumi 除本理史, un économiste de l'environnement convoqué à la branche d'Iwaki du tribunal de Fukushima, la " perte du pays natal » est quelque chose d'inédit dans l'histoire du Japon (Soeda 2017 : 119-122; Yokemoto 2016). Aussi, les avocats des requérants s'efforcent-ils de mettre en valeur cet incommensurable de la catastrophe. Mais les montants fixés par les juges sont si bas qu'ils tendent à déprécier moralement les requérants (Maeda et al. 2019 : 13-19, 67-69). 
Dans la grande majorité de ces actions collectives, les demandeurs ont saisi la justice à la fois contre Tepco et contre l'État. Ce geste revêt une signification particulière. Si les juges gardent dans l'ensemble leur distance avec le gouvernement et le Parti libéral-démocrate (PLD), ils sont néanmoins influencés par la culture politique dominante (Upham 2005 : 454; Haley 1998; Johnson 2002; Ramseyer \& Rasmusen 2003). De sorte qu'ils peuvent être réticents à fixer des niveaux d'indemnisation plus élevés lorsque l'État est mis en cause. Mais, comme les avocats l'ont fait valoir, c'est l'État qui a décidé le développement du programme électronucléaire, et ce nonobstant une menace sismique considérable; il était donc hors de question de ne pas engager la responsabilité civile de l'État (Kawai 2012; Nariwai o kaese [...] 2014 : 50-64).

\section{Le procès pénal et la responsabilité de l'État}

Dans les actions au civil, la faute doit être convertie en argent, ce qui tend à atténuer les dimensions éthiques de l'action (Jobin 2013a). Si les poursuites pénales offrent une alternative à ce piège réductionniste, elles restent rares pour les catastrophes industrielles, non seulement au Japon, mais dans le monde entier. Et lorsqu'elles peuvent être constituées, le résultat est très aléatoire. Au Japon, dans le cas de la maladie de Minamata, en 1988, deux dirigeants de la firme Chisso チッソ ont été condamnés à deux ans de prison en sursis, mais, pour les victimes, cette condamnation tardive ne présentait guère de sens (Togashi 1995; Jobin 2006).

Dans ce contexte, le procès pénal initié par 15000 habitants de Fukushima est exceptionnel. Pour convaincre le procureur d'engager les poursuites, ils se sont regroupés dans un collectif, le Gensodan 原訴団 (abréviation de Fukushima genpatsu keiji soshō shiendan 福島原発刑事訴訟支援団). Les leaders du mouvement sont une cinquantaine de militants qui, depuis le milieu des années 1980, se sont battus contre la présence massive de centrales nucléaires à Fukushima. Peu avant la catastrophe nucléaire en mars 2011, ils se préparaient à manifester contre une prolongation de dix ans d'un des réacteurs de Fukushima Daiichi ${ }^{4}$.

4. Entretien avec Muto Ruiko, représentante du Gensodan, Tokyo, 12 novembre 2019. 
L'accident nucléaire les a conduits à radicaliser et élargir leur critique. Avec leurs avocats, ils ont établi une liste d'une vingtaine de représentants de l'État et de Tepco. Les procureurs n'ont finalement retenu que trois dirigeants de Tepco et, en première instance, les juges ont conclu qu'il n'y avait pas suffisamment de preuves pour les condamner. L'affaire a été portée en appel, mais il y a peu de chance que cette décision en première instance soit infirmée (Johnson, Fukurai \& Hirayama 2020). Le procès au pénal revêt toutefois une grande importance sur le plan politique et moral, et il a permis de recueillir des preuves qui ont pu être mises à profit dans les procès au civil.

En comparaison d'autres pays, au Japon, les poursuites pénales sont rares et les acquittements encore plus. En revanche, le taux de condamnation est très élevé (99,96 \% en 2019); de sorte qu'à partir du moment où la poursuite est engagée par le procureur, on peut être à peu près certain que le(s) accusé(s) seront condamnés (Johnson 2002 : 216-218). Or, fait inhabituel, les trois dirigeants de Tepco ont été acquittés.

Pour ce type d'affaire techno-industrielle, un procès pénal est juridiquement parlant beaucoup plus difficile qu'une action au civil; il n'est donc pas surprenant qu'il ait fallu attendre juillet 2017 pour la première audience (Johnson et al. 2020). Cependant, pour le Gensodan, outre l'acquittement très inhabituel, de nombreux signes attestent d'une influence politique évidente. Ainsi, plusieurs passages du verdict prennent pour argent comptant des arguments habituellement tenus par Tepco et le gouvernement. Non seulement le procureur a écarté de l'acte d'accusation les représentants de l'État, mais encore l'affaire a ensuite été brusquement transférée de Fukushima à Tokyo, où les juges se sont montrés très hostiles envers ceux qui avaient été admis dans la salle :

Les contrôles à l'entrée étaient encore plus stricts que pour le procès de la secte d'Aum (Oumu Shinrikyō オウム真理教), comme si nous étions d'éventuels terroristes! Et bien que la salle ait 90 sièges, seule une vingtaine d'auditeurs étaient admis, par tirage au sort. Les autres sièges étaient censés servir pour la défense et les médias, mais la plupart d'entre eux sont restés vides. [...] Lors des audiences, il est parfois naturel de réagir, n'est-ce pas? Par exemple, lorsque nous avons entendu les révélations choquantes du sismologue Shimazaki Kunihiko 島崎邦彦, certains d'entre nous n'ont pu s'empêcher de laisser échapper un petit cri de surprise. En fait, c'était 
presque comme un murmure, ce n'était pas du tout bruyant. Mais le juge a menacé de faire évacuer la salle! C'était vraiment exagéré ${ }^{5}$.

On peut voir aussi dans le traitement réservé à Yamashita Kazuhiko 山下和彥 un autre indice de la partialité probable des juges. Yamashita est un ancien employé de Tepco, qui était chargé de prendre des mesures face au risque de tsunami. Dans un avis transmis à la cour, Yamashita a déclaré qu'en 2008, les trois dirigeants de Tepco avaient été informés du risque d'une vague de $15,7 \mathrm{~m}$, soit une vague légèrement supérieure à celle de $15,5 \mathrm{~m}$ qui a frappé les réacteurs en mars 2011 (Osumi 2019). Mais en juillet 2008, alors qu'ils avaient initialement approuvé des mesures de sécurité pour faire face à ce risque, les dirigeants ont mis de côté le projet pour ne pas risquer d'augmenter les craintes du public et parer à d'éventuelles manifestations locales des antinucléaires.

Avec un témoignage aussi crucial, il aurait été naturel que Yamashita figure en tête de la liste des 21 témoins assignés à comparaître. Mais les juges s'en sont tenus à une lecture orale de son avis, évitant ainsi un interrogatoire de son auteur par le procureur. Selon le Gensodan, les juges craignaient sans doute que Yamashita ne réitère ses critiques contre les dirigeants de Tepco ainsi que les hauts fonctionnaires et hommes politiques impliqués. En septembre 2013, lorsque le Premier ministre Abe avait, pour rassurer le Comité olympique, affirmé que la situation à Fukushima Daiichi était "sous contrôle ", Yamashita n'avait pas hésité à contester publiquement cette déclaration ${ }^{6}$.

Pour Johnson et al. (2020), compte tenu de l'histoire récente des procédures pénales au Japon, il est très peu probable que ce jugement soit infirmé en appel. Toutefois, comme le soulignent également les auteurs, et comme tendent à le montrer les témoignages des avocats dans les actions au civil (voir par exemple Maeda et al. 2019), les audiences lors du procès pénal ont permis de recueillir des preuves importantes, qui se sont révélées très utiles dans les actions au civil. Le procès pénal peut donc être considéré comme un élément moteur d'un vaste mouvement national pour mettre les responsables de la catastrophe en accusation et en tirer sérieusement toutes

5. Entretien avec Muto, 12 novembre 2019.

6. Idem. 
les conséquences. Toutefois, il ne faudrait pas réduire les actions collectives au civil comme de simples surgeons du procès pénal. Au contraire, on peut même dire que ces actions au civil permettent d'aboutir là où le pénal échoue : poursuivre l'État ${ }^{7}$.

En effet, les membres du Gensodan ont été très frustrés par la décision du procureur d'écarter de l'accusation les représentants de l'État. Ils ont néanmoins considéré que les trois dirigeants de Tepco retenus par le procureur n'étaient pas de simples sous-fifres, mais des acteurs clés de toute cette histoire. Par exemple, l'ancien président de Tepco Katsumata Tsunehisa 勝俣恒久 n'était-il pas familièrement surnommé «l'empereur" (Katsumata Ten.nō 勝俣天皇) par son personnel? Le Gensodan a donc décidé de continuer à suivre ce procès attentivement en coopérant avec le mouvement national des requérants engagés dans les actions au civil contre Tepco, mais aussi, élément fondamental à leur avis, contre l'État. De leur côté, les avocats des procès au civil étaient conscients qu'inclure l'État parmi les défendeurs augmenterait les difficultés, mais ils ont estimé que cela en valait la peine, car on ne pouvait laisser de côté la question fondamentale de sa responsabilité.

Au pénal, c'est le procureur qui enquête et engage les poursuites tout $\mathrm{du}$ long et, alors même que ce procès n'aurait pas eu lieu sans une large initiative des habitants de Fukushima touchés par la catastrophe, ceux-ci ne peuvent qu'assister passivement aux audiences, qui plus est, en nombre très limité. À l'inverse, au civil, les victimes interviennent directement dans la construction du recours et, pendant les audiences, même si leur rôle est contraint par la procédure et la stratégie adoptée par leurs avocats, fondamentalement, ce sont les victimes qui sont à l'origine de l'action et la portent jusqu'à son terme : si pour une raison quelconque, les demandeurs décident de se retirer, le procès prend fin. Ainsi, dans le cas de Fukushima, les victimes ont pu se constituer en collectif de requérants, avec l'aide de leurs avocats, afin de questionner à leur façon le rôle de l'État dans la chaîne de décisions qui a provoqué la catastrophe nucléaire ainsi que les diverses politiques adoptées depuis lors.

7. Entretiens séparés avec des représentants de Gensoren et Gensodan, Tokyo, novembre 2019. 


\section{Les procès en nom collectif comme mouvement social}

Que ce soit au civil ou au pénal, tous ces procès relatifs à la catastrophe de Fukushima s'inscrivent dans une histoire et une dynamique collectives. À bien des égards, on peut considérer l'ensemble de ces procès comme un mouvement social à part entière. Les procès servent d'amorce fédératrice à une ambition plus vaste, soit ce que les avocats et les requérants engagés dans ce type de bataille juridique nomment couramment un " mouvement de procès " (soshō undō 訴訟運動). Or, ce mouvement vient d'assez loin et il innerve tout le Japon contemporain. Voyons-en brièvement la genèse et de quelle façon les procès de Fukushima l'infléchissent.

Lorsqu'on parle de "mouvement de procès ", le premier héritage qui vient à l'esprit, ce sont les procès en nom collectif entrepris par les victimes de la pollution industrielle (kōgai soshō 公害訴訟). À l'ère Meiji, les paysans d'Ashio touchés par la pollution de la mine de cuivre du zaibatsu 財閥 Furukawa 古河 avaient envisagé cette possibilité, sans donner suite (Pitteloud 2019 : 132-133). Ce n'est qu'après la seconde guerre mondiale que les mouvements contre la pollution ont commencé à recourir plus fréquemment à une approche judiciaire, à commencer par les «quatre grands procès de la pollution " (yondai kögai saiban 四大公害裁判) pour les victimes de la maladie de Minamata à Kyūshū et à Niigata, l'empoisonnement au cadmium à Toyama et l'asthme de Yokkaichi. Ces procès étaient "grands", moins par le nombre de requérants que par la violence de ces pollutions, le nombre potentiel de victimes, et pour leur nouveauté (entre 1967 et 1973).

À partir des années 1980, le Parti communiste japonais a lancé d'autres procès avec, cette fois, des milliers de requérants impliqués; il s'agissait surtout des victimes de la pollution atmosphérique vivant à proximité de zones industrielles telles que Kawasaki ou Kitakyūshū (Jobin 2006; Nichibenren 2010). Parmi d'autres grands mouvements de victimes engagés dans des batailles juridiques collectives, citons aussi celui des mineurs atteints de la silicose ou d'autres formes de pneumoconiose (Thomann 2009, 2013), les victimes de l'amiante (Jobin 2013a), ainsi que les survivants de la bombe atomique (Genbakushō nintei shūdan 2011).

Tous ces procès constituent un vaste répertoire d'actions collectives et de références juridiques. Tandis que les juristes ont tendance à mettre l'accent sur les résultats positifs obtenus grâce à ces luttes (Nichibenren 
2010; Shimabayashi 2010), d'autres analystes mettent aussi en lumière les tensions entre différents acteurs comme les avocats et leurs clients, les syndicalistes et les écologistes (Upham 1987; George 2001; Jobin 2006).

Ces procès en nom collectif (dantai soshō 団体訴訟) visent à offrir une réparation pour une communauté de victimes qui ne s'arrête pas aux seuls requérants. En ce sens, ils partagent le même but que leurs équivalents européens (ou dans d'autres pays asiatiques comme Taiwan et la Corée), ou encore les class actions américaines. Toutefois, d'un pays à l'autre, ou d'un mouvement à l'autre, les motivations des avocats et de leurs clients sont assez variables. Ainsi, aux États-Unis, lorsque les avocats peuvent faire valoir des punitive damage (dommages et intérêts punitifs), les réparations peuvent atteindre des sommes astronomiques, ce qui offre aux victimes une véritable réparation et une marque de respect, mais tend à transformer ces procès en " vache à lait " pour des cabinets d'avocats dont les pratiques agressives choquent leurs homologues japonais. Et cet environnement retarde ou bloque les changements législatifs logiquement attendus; ce fut le cas des centaines de milliers de litiges relatifs à l'amiante sans pour autant, comble du paradoxe, bannir totalement son usage (Jasanoff \& Perese 2003).

À l'inverse, au Japon, comme nous l'avons vu, les indemnités accordées par les tribunaux sont faibles. Les avocats qui s'engagent aux côtés des requérants ne sont donc a priori pas motivés par la perspective d'honoraires juteux (Steinhoff 2014). Les motivations sont plutôt d'ordres politique et moral et elles sont relayées par une chaîne de militants associatifs, des syndicalistes, des médecins et différents chercheurs qui trouvent appui auprès de leurs réseaux d'avocats. Ces derniers sont prêts à s'investir sur le long terme et, à la rigueur, quelles que soient les chances de succès; leur conscience professionnelle et une dynamique de groupe (l'interaction avec les requérants et leurs confrères avocats) font le reste. Si les sommes obtenues s'avèrent beaucoup plus faibles qu'aux États-Unis (mais comparables à la France ou d'autres pays d'Asie comme Taiwan et la Corée), cet environnement a l'avantage d'encourager indirectement la formation de mouvements sociaux qui ne limitent pas leur action à l'enceinte du tribunal, mais visent aussi, si nécessaire, à modifier les politiques publiques et la législation.

Ainsi, beaucoup d'avocats engagés dans ces luttes sont membres du Parti communiste japonais (Nihon kyōsantō 日本共産党); ou bien ils en sont idéologiquement proches, ou bien ils sont familiers des réseaux politiques 
ou associatifs de ce qui reste de l'ancien Parti socialiste (Nihon shakaitō 社会党), c'est-à-dire le Parti social-démocrate (Shamintō 社民党) et l'ancienne aile gauche du Parti démocrate du Japon (Minshutō民主党), rebaptisé Parti démocrate constitutionnel (Rikken minshutō 立憲民主党). Si, au Parlement, ces partis sont numériquement dominés par le PLD, leurs réseaux demeurent socialement très actifs et influents dans les médias.

Une autre caractéristique importante de ces affaires techno-industrielles est le long délai d'attente pour que les décisions soient rendues, et lorsqu'une bataille se termine, une autre commence, d'où l'impression d'une histoire sans fin. Le cas des procès relatifs à la maladie de Minamata est emblématique; des années 1960 jusqu'à aujourd'hui, on en compte plus d'une trentaine contre les firmes Chisso (à Kyūshū) et Shōwa denkō 昭和電工 (à Niigata), et aussi contre l'État, avec des dizaines de milliers de réquérants, pour l'essentiel, les laissés pour compte du système d'indemnisation instauré par l'État au début des années 1970 (Jobin 2014). Après mars 2011, les militants et chercheurs engagés au côté des victimes de la maladie de Minamata ont eu à cœur de partager avec les victimes de Fukushima leurs décennies d'expérience de lutte contre l'État et les industries polluantes (Genbakushō nintei soshō Kumamoto bengodan 2011).

Dans le cas de Fukushima, un nombre équivalent de procès ont été intentés, et ce, en moins de dix ans. Le premier a démarré dès 2012, soit très peu de temps après la catastrophe. Pourquoi une telle différence? Relève-t-elle par exemple d'un " tournant juridique » du mouvement antinucléaire? Je postule au contraire que le nombre et le lancement rapide de ces procès s'appuient sur un héritage de "mouvements de procès ". Si la composante antinucléaire est présente dans les procès de Fukushima, leur portée idéologique dépasse l'opposition à une forme particulière d'énergie ou d'industrie.

Malgré l'augmentation des maladies de la thyroïde après la catastrophe, pour l'heure, il n'y a pas encore d'action collective pour demander un pretium doloris ou un préjudice d'anxiété par rapport au risque de cancer. Les actions en cours présentent néanmoins de nombreuses similitudes avec les batailles juridiques menées par les victimes de la bombe atomique, soit un total de vingt-deux procès collectifs pour contester les critères ultra restrictifs établis par l'État pour reconnaître les symptômes de la bombe. La plupart des tribunaux se sont prononcés en faveur des demandeurs. Après mars 2011, leurs avocats ont jugé utile de réunir les preuves qu’ils avaient 
présentées aux juges, anticipant les besoins juridiques des victimes de Fukushima à l'avenir (Genbakushō nintei shūdan 2011).

Par ailleurs, depuis la fin des années 1970, des militants syndicaux et des associations de surveillance du nucléaire ont engagé une vingtaine de procès et des batailles administratives pour des cas de leucémie et autres maladies graves contractées par les travailleurs des centrales nucléaires (Jobin 2013b). D'autres actions semblables entreprises après mars 2011 participent pleinement au mouvement des procès de Fukushima, et leur signification est très importante pour tout ce qui touche aux normes d'exposition (Iida 2016; Jobin 2019).

Une autre référence importante pour les procès de Fukushima, réside dans les nombreux litiges contre les centrales nucléaires qui ont également commencé à la fin des années 1970. L'avocat Kaido Yūichi 海渡雄一, un des leaders de ce mouvement, en a recensé un total de seize. Avant 2011, il s'agissait d'empêcher l'ouverture ou de faire arrêter une centrale ou un réacteur; in fine, tous ces procès ont été perdus, dont une dizaine devant la Cour suprême. Seules deux victoires provisoires ont pu être arrachées en appel : l'une concernait un réacteur de la centrale de Shika (département d'Ishikawa), et l'autre, le réacteur Monju (département de Fukui), dont les ambitions d'innovation technologique se sont soldées par un gouffre financier d'un trillion de yens. Pour Kaido, ces décisions judiciaires marquent une véritable faillite du système judiciaire japonais, et en particulier de la Cour suprême; comme les risques de séisme et de tsunami jouaient un rôle central dans ces recours, on peut même dire que la Cour suprême porte une lourde responsabilité pour n'avoir pas su prévenir la catastrophe de Fukushima (Kaido 2011; Isomura \& Yamaguchi 2016).

En comparaison, les actions engagées après mars 2011 contre les projets du gouvernement de redémarrer les centrales nucléaires ont rencontré un plus grand succès. Dès juillet 2011, Kaido et Kawai Hiroyuki 河合弘之 ont réuni un groupe de 170 avocats pour établir des recours administratifs appelés " mesures provisoires de suspension de l'exploitation " (unten sashitome karishobun 運転差し止仮処分) dont ils ont acquis une longue pratique. Hormis quatre centrales (Higashidōri, Onagawa, Fukushima Daiichi et Daini), ce mouvement de procès concerne l'ensemble du parc nucléaire japonais. En avril 2015 par exemple, le tribunal de district de Fukui s'est prononcé contre le redémarrage des réacteurs numéro 3 et 4 de la centrale nucléaire de Takahama. Une décision de justice visant à suspendre 
l'exploitation n'a pas de pouvoir coercitif sur les compagnies d'électricité qui exploitent les centrales, mais elle envoie un avertissement très clair à la population locale et au gouverneur (Kawai 2015).

Ce vaste mouvement social des procès de Fukushima comporte donc clairement une dimension politique. Pour autant, il ne semble pas trop souffrir des logiques partisanes. De tels mouvements se sont longtemps trouvés divisés entre socialistes et communistes (avec des organisations différentes, comme la Gensuikin 原水禁 et la Gensuikyō 原水協 dans le cas du mouvement antinucléaire). Même si des tensions subsistent encore aujourd'hui, la disparition du Parti socialiste japonais au milieu des années 1990 a atténué ces clivages. Ainsi, bien que la coalition nationale Gensoren (qui réunit l'ensemble des requérants pour les procès de Fukushima au civil) soit proche du Parti communiste, elle coopère activement avec le Gensodan (à l'initiative du procès pénal), qui a pourtant plus d'affinités avec le réseau de l'ancien Parti socialiste. De façon plus marginale, le Gensoren coopère aussi avec Yamamoto Tarō 山本太郎 qui, à l'issue de la catastrophe, avait fondé le parti indépendant Reiwa shinsengumi れいわ新選組.

\section{Conclusion}

Les actions collectives engagées par les victimes de la catastrophe de Fukushima s'inscrivent dans une culture de contestation qui légitime le procès en tant que mouvement social. Les avocats et requérants engagés dans une trentaine d'actions au civil contre Tepco et l'État et les cadres de Tepco ont formé une coalition nationale qui travaille de concert avec les associations à l'origine du procès pénal. Malgré l'acquittement en première instance des trois cadres de Tepco, le procès pénal a insufflé une dynamique qui interroge en profondeur les causes de l'accident nucléaire; après que le procureur a écarté la responsabilité de l'État, la question a été relayée par les procès au civil. Et malgré les difficultés que cela implique, dans la majorité des procès au civil, les requérants ont engagé non seulement la responsabilité de Tepco, mais aussi celle de l'État. Plusieurs tribunaux, dont une Cour d'appel, ont confirmé la responsabilité de l'État dans la catastrophe et condamné l'insuffisance de l'aide apportée aux familles déplacées. Bien que, jusqu'à présent, les montants d'indemnisation fixés par les juges soient très faibles, ce problème ne semble pas freiner la motivation des requérants. 
Les procès de Fukushima prennent appui sur un grand nombre de procès antérieurs, notamment les batailles juridiques contre la construction ou l'extension des centrales nucléaires et celles des hibakusha pour contester les critères de reconnaissance des symptômes de la bombe atomique. Les procès des malades de Minamata et d'autres catastrophes industrielles, ainsi que les batailles administratives aux côtés des ouvriers du nucléaire atteints de leucémies, constituent d'autres sources d'inspiration des mobilisations en cours. À l'instar de ces antécédents, les procès de Fukushima ont d'abord surgi d'initiatives locales, à peu près partout où se sont trouvées des personnes déplacées par la catastrophe, avant de converger pour former une alliance nationale.

Bien que les procès de Fukushima n'aient pas fondamentalement remis en cause le contrôle du pouvoir par le PLD, ce vaste mouvement social réunissant différents procès $(s o s h \bar{o}$ undō), et dont l'initiative revient aux victimes de la catastrophe, a contribué à empêcher le gouvernement de redémarrer ses réacteurs nucléaires. Comme le soulignent Steinhoff et al. (2014), et comme cela est évident dans le procès pénal contre les trois cadres de Tepco, une défaite devant les tribunaux ne signifie pas nécessairement une défaite pour le mouvement social dans son ensemble. À tout le moins, un procès en nom collectif nourrit une dynamique de combat et de solidarité; il comporte aussi ce qu'Eric Seizelet (2009) nomme « une fonction tribunicienne " qui contribue en l'occurrence à faire connaître la situation économiquement difficile de nombreux requérants. Mais le contraire peut également être vrai : une victoire devant les tribunaux ne garantit pas toujours que le mouvement atteigne ses objectifs, ni qu'il contribue à une réforme des politiques publiques ni un changement social en profondeur. Quoi qu'il en soit, ces procès mériteraient donc une plus grande attention des chercheurs spécialistes du Japon contemporain. 


\section{Bibliographie}

\section{Foote Daniel 2014}

«Cause Lawyering in Japan: Reflections on the Case Studies and Justice Reform », in Steinhoff Patricia G. (dir.), Going to Court to Change Japan: Social Movements and the Law in Contemporary Japan, Ann Arbor, The University of Michigan Press : 165-180.

\section{Fukurai Hiroshi, Hirayama Mari}

\& JoHnson David 2020

"Reflections on the TEPCO Trial:

Prosecution and Acquittal after Japan's

Nuclear Meltdown », The Asia-Pacific

Journal, 18 (2), n 1.

Genbakushō nintei shūdan soshō kirokushū kankō iinkai 原爆症認定集 団訴訟·記録集刊行委員会 (Collectif des procès pour la reconnaissance des symptômes de la bombe atomique) 2011

Genbakushō nintei shūdan soshō tatakai no kiroku 原爆症認定集団訴訟たたかいの 記録 (Archives du Collectif des procès pour la reconnaissance des symptômes de la bombe atomique), Tokyo, Nihonhyōronsha 日本評論社.

\section{Genbakushō nintei soshō Kumamoto} bengodan 原爆症認定訴訟熊本弁護団 (Procès pour la reconnaissance des symptômes de la bombe atomique : équipe des avocats de Kumamoto) 2011

Minamata no kyōkun o Fukushima e 1: Minamatabyō to genbakusho no keiken o fumaete 水俣の教訓を福島へ〜水俣病と
原爆症の経験をふまえて (De Minamata à Fukushima : l'expérience de la maladie de Minamata et des symptômes de la bombe), Kumamoto, Kadensha 花伝社.

GEORGE Timothy 2001

Minamata. Pollution and the Struggle for Democracy in Postwar Japan, Cambridge, Harvard University Press.

\section{HaLey John 1978}

"The Myth of the Reluctant Litigant ", Journal of Japanese Studies, 4 (2) : 359-90.

HaLEY John 1991

Authority without Power: Law and the Japanese Paradox, New York, Oxford University Press.

\section{HALEY John 1998}

The Spirit of Japanese Law, Athens, University of Georgia Press.

\section{IIDA Katsuyasu 飯田勝泰 2016}

«Fukushima genpatsu ni okeru hibaku rōdōmondai no genjō to kadai » 福島 原発における被ばく労働問題の現状と課題 (Situation actuelle et questions relatives au travail sous rayonnement dans les centrales nucléaires de Fukushima), Rōdō hōritsu junpō 労働法律旬報 (Revue de droit du travail), 1872, septembre : 50-58.

\section{IsomURA Kentarō 磯村健太郎}

\& YAMAGUCHI Eiji 山口栄二 2016 Genpatsu ni idonda saibankan 原発に挑ん だ裁判官 (Les juges mis en question par les centrales nucléaires), Tokyo, Asahi shimbunsha 朝日新聞社. 
Jasanoff Sheila \& Dogan Perese 2003

«Welfare State or Welfare Court:

Asbestos Litigation in Comparative

Perspective ", Journal of Law and Policy, 12 : 619-637.

\section{JoBin Paul 2006}

Maladies industrielles et renouveau syndical au Japon, Paris, Éditions de I'EHESS.

\section{JoBIN Paul 2013a}

«The Criminalization of Industrial Disease: Epidemiology in a Japanese Asbestos Lawsuit ", in PECKHAM Robert (dir.), Disease and Crime: Social Pathologies and the New Politics of Health, Londres, Routledge : 170-191.

\section{JoBIN Paul ジョバン・ポール 2013b} « 3.11 Jiko ikō no hōshasen bōgo »

3.11 事故以降の放射線防護 (La radioprotection après l'accident nucléaire de Fukushima), Ōhara shakai mondai kenkyūjo zasshi 大原社会問題 研究所雑誌, 8 (658) : 14-30.

\section{JobIn Paul 2014}

« Beyond Uncertainty: Industrial Hazards and Class Actions in Taiwan \& Japan », in Lıu Ts'ui-jung (dir.), Environmental History in East Asia: Interdisciplinary Perspectives, Londres, Routledge : 339-382.

\section{JoBin Paul 2017}

«"Nuclear Gypsies" in Fukushima Before and After 3.11. ", in MACDowELL Laurel (dir.), Nuclear Portraits: People, Communities and the Environment, Toronto, University of Toronto Press : 274-311.

\section{JoBin Paul 2019}

"Some 40 Years to Clean Up Fukushima: A View from Ongoing Court Battles », Georgetown Journal of Asian Affairs, 4 (2) : 73-81.

\section{JoBIN Paul 2020}

"The Fukushima Nuclear Disaster and Civil Actions as a Social Movement ", The Asia Pacific Journal: Japan Focus, $1^{\mathrm{er}}$ mai.

\section{JoBIN Paul 2021}

« Nuclear Labor, Its Invisibility, and the Dispute over Low-Dose Radiation », in Cleveland Kyle, Knowles Scott Gabriel \& SHINEHA Ryuma (dir.), Legacies of Fukushima: 3.11 in Context, Pennsylvania, University of Pennsylvania Press : 145-156.

\section{Johnson David 2002}

The Japanese Way of Justice: Prosecuting Crime in Japan, Oxford, Oxford University Press.

\section{KAIDo Yũichi 海渡雄一 2011}

Genpatsu soshō 原発訴訟 (Les centrales nucléaires en procès), Tokyo, Iwanami shoten 岩波書店.

\section{KAIDO Yūichi 2018}

Töden keiji saiban de akiraka ni natta koto 東電刑事裁判で明らかになったこと (Ce que le procès pénal contre Tepco a mis en lumière), Tokyo, Sairyūsha 彩流社.

\section{Kawal Hiroyuki 河合弘之 2012}

Tōden kabunushi daihyō soshō : genpatsu jiko no sekinin o tou 東電株主代表訴訟 一原発事故の責任を問う (Procès des représentants des actionnaires de Tepco pour leur responsabilité dans l'accident 
nucléaire), Tokyo, Gendai jinmonsha 現代人文社.

\section{Kawal Hiroyuki 2015}

Genpatsu soshō ga shakai o kaeru 原発 訴訟が社会を変える (Les procès contre les centrales nucléaires changent la société). Tokyo, Shūeisha 集英社.

\section{KIKUCHI Tsutomu 菊池功 2020}

"Hisai-chi no "mei" to "an" hōdō tsuzukeru » 被災地の「明」「暗」報道続ける (Continuer à rendre compte du clairobscur des zones dévastées), Asahi shinbun 朝日新聞, 11 mars.

\section{KoJIMA Rina 2020}

«Reconstruire dans l'après-Fukushima: responsabiliser et vulnérabiliser par le risque ", thèse de doctorat en sociologie, université Paris-Est.

MAEDA Akira 前田朗 et al. 2019

Fukushima genpatsu shūdan soshō no hanketsu o megutte : minshū no shiza kara 福島原発集団訴訟の判決を巡って一民 衆の視座から (Les jugements des procès collectif contre la centrale nucléaire de Fukushima : le point de vue du public), Tokyo, Dokushojin 読書人.

« Nariwai o kaese, chiiki o kaese!» Fukushima genpatsu saiban genkokudan bengo-dan「生業を返せ、地域を返 せ!」福島原発裁判原告団·弁護団 (Les plaignants et les avocats du procès nucléaire de Fukushima « Rendeznous notre travail, rendez-nous notre région ») 2014

Anata no Fukushima genpatsu soshō: minna shite «nariwai o kaese, chiikio kaese» あなたの福島原発訴訟一みんなして 「生業を返せ、地域を返せ!」(Votre procès de Fukushima), Kyoto, Kamogawa shuppan かもがわ出版.

\section{Nihon bengoshi rengōkai kōgai taisaku/kankyō hozen iinkai 日本弁 護士連合会公害対·策環境保全委員会} (Barreau des avocats japonais : Comité de contrôle de la pollution industrielle et de protection de l'environnement) 2010

Kōgai/kankyō soshō to bengoshi no chōsen 公害·環境訴訟と弁護士の挑戦 (La pollution industrielle et les litiges environnementaux : un défi pour les avocat), Kyoto, Hōritsu bunkasha 法律 文化社.

Osumı Magdalena 2019

«Former Tepco Executives Found Not Guilty of Criminal Negligence in Fukushima Nuclear Disaster », Japan Times, 19 septembre.

Pitteloud Cyrian 2019 «L'Affaire d'Ashio : pollution minière et expertise environnementale dans le Japon moderne ", thèse de doctorat, université de Genève.

Ramseyer Mark \& Rasmusen Eric 2003 Measuring Judicial Independence: The Political Economy of Judging in Japan, Chicago, University of Chicago Press.

\section{Ribault Thierry 2019}

«Resilience in Fukushima: Contribution to a Political Economy of Consent ", Alternatives: Global, Local, Political : 1-25. 
SATō Yoshiyuki 佐藤嘉幸 2020

«Satō Yoshiyuki-shi ga kiku datsu

genpatsu shirīzu “Kaido Yūichi bengoshi-

hen : toden keiji saiban no hanketsu no

ayamari o tettei hihan suru"» 佐藤嘉幸氏

が聞く脱原発シリーズ《海渡雄一弁護士編:

東電刑事裁判の判決の誤りを徹底批判する》

(Série d'entretiens de Satō Yoshiyuki sur

la dénucléarisation : I'avocat Kaido Yūichi

dresse une critique radicale des erreurs

commises par le procès pénal contre

Tepco), Dokushojin web (dokushojin.com).

\section{Scoccimarro Rémi 2016}

« 11 mars 2011 : de la vie en

préfabriqués à l'assignation à

resilience ", in DOUmEt Christian \& FERRIER

Michaël (dir.), Penser avec Fukushima,

Paris, Cécile Defaut : 133-159.

\section{Seizelet Eric 2009}

« Le Japon et la Cour pénale

internationale : enjeux politiques

et mémoriels », Droit et cultures, 58 :

85-108.

\section{SoEDA Takashi 添田孝史 2017}

Tōden genpatsu saiban : Fukushima genpatsu jiko no sekinin o tou 東電原発裁 判—福島原発事故の責任を問う (Le procès de la centrale nucléaire de Tepco : exiger la responsabilité de l'accident nucléaire de Fukushima), Tokyo, Iwanami shoten.

\section{SHIRAI Satoshi 白井聡 2015}

Fukushima genpatsu jiko higai bengodan, Fukushima o kirisuteru nodesu ka: « 20 mirishïberuto » junin-ron hihan 福 島原発事故被害弁護団, 福島を切り捨て るのですか一“20ミリシーベルト”受忍論 批判 (Vous voulez couper les vivres à
Fukushima? Une critique de la thèse des « 20 millisieverts »), Kyoto, Kamogawa shuppan.

Steinhoff Patricia (dir.) 2014

Going to Court to Change Japan: Social Movements and the Law in Contemporary Japan, Ann Arbor, University of

Michigan.

\section{Thomann Bernard 2009}

« L'hygiène nationale, la société civile et la reconnaissance de la silicose comme maladie professionnelle au Japon (18681960) ", Revue d'histoire moderne \& contemporaine, 56-1 : 142-176.

\section{Thomann Bernard 2014}

«Les victimes invisibles de la pneumoconiose dans les mines de charbon au Japon », in JUDiTH Rainhorn (dir.), Santé et travail à la mine, $x I X^{e}-X X I^{e}$ siècles, Lille, Presses universitaires du Septentrion : 151-172.

\section{TogASHI Sadao 冨樫貞夫 1995}

Minamatabyō jiken to hō 水俣病事件と法 (La maladie de Minatamata et la loi). Fukuoka, Sekifūsha 石風社.

\section{UPHAM Frank 1976}

"Litigation and Moral Consciousness in Japan: An Interpretive Analysis of Four Japanese Pollution Suits ", Law \& Society Review, 10 (4) : 579-619.

\author{
UPHAM Frank, 1987 \\ Law and Social Change in Postwar Japan, \\ Cambridge, Harvard University Press.
}


UPHAM Frank 2005

«Political Lackeys or Faithful Public

Servants? Two Views of the Japanese

Judiciary ", Law \& Social Inquiry, 30 (2) :

421-455.

\section{Xuan Bien Do 2019}

«Fukushima Nuclear Disaster

Displacement: How Far People Moved

and Determinants of Evacuation

Destinations ", International Journal of

Disaster Risk Reduction, 33 : 235-252.

Yокемото Masafumi 除本理史 2016

Kōgai kara Fukushima o kangaeru : chiiki

no saisei o mezashite 公害から福島を考

える一地域の再生をめざして (Fukushima

au prisme de la pollution industrielle :

vers une régénération régionale), Tokyo, Iwanami shoten.

Yокемото Masafumi 2020

«Fukushima genshiryoku hatsudensho

jiko ni okeru higaisha shūdan soshō

no dōkō » 福島原子力発電所事故におけ

る被害者集団訴訟の動向 (Évolution des

procès collectifs relatifs à l'accident de la centrale nucléaire de Fukushima), Keihei kenkyū 経営研究, 71 (3) : 37-47. 